\title{
AN ARCHITECTURE-BASED TECHNIQUE TO MOBILE CONTACT RECOMMENDATION FOR EMERGENCY SITUATION IN NIGERIA
}

\author{
B. I. Akhigbe ${ }^{1,}$, R. N. Ikono' ${ }^{2}$ A. O. Ejidokun ${ }^{3}$, S. O. Aderibigbe ${ }^{4}$ and B. S. Afolabi ${ }^{5}$ \\ 1,2,3,5INFORMATION STORAGE AND RETRIEVAL RESEARCH GROUP, DEPT. OF COMPUTER SCIENCE \& ENGINEERING, OBAFEMI \\ AWOLOWO, UNIVERSITY, ILE-IFE, OSUN STATE. NIGERIA. \\ 4DEPartment of Computer Science, Lagos State Polytechnic, IKoRodu, Lagos STATE. NIGERIA \\ E-mail addresses: ${ }^{1}$ benplus1@gmail.com,2rhoda_u@yahoo.com,3 gbengskul@gmail.com, \\ 4 aderibigbe2000@gmail.com, 5 afolabib@gmail.com
}

\begin{abstract}
Smart technologies such as smart phones, iPad and Tablets are ubiquitous in today's society. They possess increasing computing and storage potentials. Thus, emerging as a dominant computing platform for different kinds of end-users. However, these technological possibilities have not been fully explored for emergency situations where close relatives must be contacted. This paper therefore presents an Emergency Contact Recommendation Model (ECRM) that was implemented into an emergency contact recommendation system. An architectural based approach was employed to highlight the contribution this paper made to extant knowledge. The leveraged of the Dust miner algorithmic technique, the direct discriminative pattern mining, and the Bayesian Inference Network technique were used to formulate the ECRM. The ECRM was implemented using the Java development and android tool kit. The model demonstrated commendable capabilities - considering the foregoing techniques when compared with what obtains in literature- to make useful recommendation in emergency situation(s) after implementation.
\end{abstract}

Keyword: Smart technologies, Emergency contact recommendation model, Emergency contact recommendation system, soft-computing technology, Data mining

\section{INTRODUCTION}

Smart Computing Technologies (SCT) such as iPhones, iPad, smartphones and Tablets are ubiquitous in today's society. Despite being small, portable, and easy to use, these devices are equipped with computing power that rivals larger and less portable desktop computers. Notably, these devices are highly configurable and they allow downloadable applications (or apps) that are unlimited in scope [1]. Even experts had predicted that by the end of 2014, the number of smartphones in circulation will exceed the number of personal computers in use [2]. This is because of the crave for SCT to satisfy the almost endless need to do e-mail, browse, enjoy $3 \mathrm{G}$ and $4 \mathrm{G}$ data services, and also have compelling user experiences in platforms such as Google Maps, Facebook, YouTube(and other social media platforms) on the fly [3]. It is interesting to note that all mobile phones are not smartphones. But, they all have the same ability to store the names and phone number(s) of Relatives, Friends or other Close Acquaintance (RFoCA). Aside being able to store the name of contacts and their phone numbers, there are other details that can be stored [4]. For instance, every Smartphone has the ability to keep the Log of Call Transactions (LoCTs) of callers and callers over a period of time depending on the phone make and the operating system it uses. Interestingly, the LoCTs of phones including that of Tablets and iPads are one resource which has not been taken advantage of in emergency situations.

Emergency situations do result from situation(s) that require urgent attention. For instance, if there was a ghastly vehicle accident, or a fire outbreak in a commercial area or a bomb attack there will be the situation of emergency. In such emergency situation the belief system in Nigeria that is culturally and religiously inclined makes it very likely to always have willing Nigerians (aside the possibility of having government agencies) come to the aid of victims in a rescue operation. During such operations rescuers do have access to victim's mobile phone, which has LoCTs and other information that are mineable. However, the question is; how would rescuers know which one of the contact numbers (out of the tens of mobile contact numbers or LoCTs on the phone) that will result to a 
useful contact. By useful contact we mean a contact number of a close relative or acquaintance who can take responsibility for the situation at hand. One can argue that, while not call the last dialled number by the victim or the last number he/she received before the emergency situation. For sure, this is a valid argument; but in a situation where subscribers receive arbitrary calls even from GSM service provides, how can one be sure that the last call is not from service provides. Usually their calls cannot be returned. An apps approach is definitely needed to help rescuers who have access to victims' mobile phone in emergency situation(s) to be able to know which contact number(s) to call in order to get across to RFoCA. The challenge of which person RFoCA to contact in an emergency situation can be really frustrating. Similarly, findings have shown that when patients are brought to the Hospital; in the emergency room the consent of RFoCA are needed before very meaningful attention can be given to victims [5].

It is often traumatic for rescuers on the scene of an accident, when they realize that victims have their phones intact, and they cannot use them to make useful contact(s). Interestingly, seamless communication between rescuers and victims' RFoCA still continue to be elusive In Cases of Emergencies (ICE) in Nigeria even though smartphones are carried about by several Nigerians. Traditionally, the concept of ICE had allowed people to program emergency contact information into the contact list of their mobile phone under the acronym "ICE". The motivation for ICE came from the understanding that emergency service personnel and Hospital staff should be able to quickly contact any person by simply dialling the number stored as ICE [6]. Though, the ICE concept was endorsed in 2013 by the Federal Road Safety Commission (FRSC) in Nigeria [7], a lot is still needed to be done to harness the good of the concept.

The thinking in this paper is that the concept of ICE can be taken further using the leverage of Information and Communication Technology (ICT). For instance, Intelligent Software Application(s) (ISAs) drawing on soft-computing techniques can be used to harness the resource(s) ICT offers. When ISAs are installed on a victim's phone, they can recommend to rescuers, which contact to call that will prove useful when they make recourse to it during an emergency situation. To achieve this, this paper adopted the Bayesian Network technique (BNt) [22] among other soft-computing technique like the artificial neural network technique [21], along with other synergistic techniques. This is because the BNt is easily implemented as a simple look-up with decision tree and table, and it is intrinsically fast. Its reasoning is based on a real-world model, with a thorough understanding of the processes involved, rather than just a mere association of data and assumptions [23].

The goal of this paper is to present a model approach to the development of Emergency Contact Recommendation System (ECRS) by extending the concept of ICE as theorized by Bob Brotchie [6]. To achieve the foregoing objective, the following specific objectives were addressed. First, we formulated a model with probabilistic properties to handle (i) optimized probabilistic inference in order to unravel issues of uncertainty, and utilize expert's pre-knowledge, and (ii) the complex issue of classification. In this work expert's pre-knowledge was assumed as the most frequently called number based on the LoCTs, and the presence of a group of numbers that were designated as family members or close acquaintance. Secondly, we implemented the model into the ECRS, and thirdly the ECRS was tested in order to ascertain its usability. This paper contributed a data mining technique that will be useful for recommending valuable contacts in crisis or emergency situations. The paper is further organized as follows; Section 2.0 contains the paper's literature review and concept presentation; section 3.0 contains the methodology employed for the proposed ECRS development starting from model's formulation; and section 4.0 contains the ECRS implementation. In section 5.0 the paper's conclusion is presented.

\section{THEORETICAL CONCEPTION}

Recommendation is part of everyday life. This concept has been modelled into software systems that are known as Recommender Systems (RSs) to help users make choices. Currently, RS use techniques that include collaborative filtering, content-based filtering, knowledge-based and hybrid approaches [8]. Recently, the successes achieved in the use of RSs as a result of the foregoing techniques has encouraged their introduction into contact and callee recommendation [8, 24]. However, to use RSs some level of intelligence must be introduced into it. This means data must be extracted and mined in order to unravel useful patterns from which inferences are made. The mining of data entails the analysis of (often large) observational data sets. The goal is to find unsuspecting relationship(s) that are summarized in novel ways that are both understandable and useful to the data owner [9].

The concept of recommendation has helped to classify data into useful categories; and cell phones are available that can support more tasks aside phone calls and texting. The use of smartphones has also reached an alltime high. With their level of computational and storage capability, there exist lots of data from them. These data can be classified from logs and sensors of smartphones 
from which patterns of users' behaviour can be mined. Enigmatically, despite the diverse functionalities that are available on smartphones, recommending a contact person during emergency situation is still a huge challenge. In Zhuang et al. [24], the RS developed uses mainly calendar data to provide contact recommendation, but not in emergency situation.

In emergency situations smartphone currently allow users to only sequentially search through its LoCTs and the list of the contacts on it. This is usually time consuming. The fact that there is no known Emergency Contact Recommendation Model (ECRM) (to the best of our knowledge), let alone an app that is implemented based on the technique(s) offered by the model further exacerbate the foregoing challenge. This makes the contribution in this paper novel to the domain of emergency contact recommendation using softcomputing techniques. The emergency contact model presented in this paper was developed into an app, such that on its activation on a victim's phone relevant contact details will be updated. This update would take advantage of the myriads of useful relationships that exist in the contact details.

\section{RELATED WORK}

There has been some work on mobile context awareness that extends the use of the address book on phones. This is reminiscent of the digital traces users of smartphones leave behind considering the volume of call transactions they carry out on a daily basis. In [10] the address book of mobile phones was extended for easy update. The researcher's work drew on the concept of context awareness information; though this was done manually. The challenge with the manual approach is such that updating the address book - on a regular basis - poses a lot of bottlenecks, some of which are very tedious on users. Schmidt et al. [11] took the foregoing success further by incorporating the concept of availability and presence to determine who a user may probably call considering (the right) time and place. For, [12] and [20] availability was considered from different perspectives. But, the main focus of their work was on how not to interrupt seamless communication with should be contact persons.

Interestingly, other more recent researches had come quite close to providing useful solution when there is the need for contact to be made to RFoCA. For instance, [13] proposed an emergency contact model that used social status, location information since they reside as calendar items on mobile phones. The model used these parameters to determine the most appropriate third party to call in an emergency situation. However, information about a third party appointments/schedules might not be saved on a calendar (on a regular basis) in a victim's phone. As a result, it may be difficult to mine a third party contact to determine the degree of closeness/relationship with the victim and other members of the contact list. In [14], the task of how to mitigate the challenge of determining the degree of closeness of relationship among the members of the contact list of a phone was initiated. The model proposed used a probabilistic social mining approach. The level of probabilism exhibited by the model drew on the Bayesian technique. In a related effort the work of [3] like the others reviewed so far only considered scenarios that are social in context, and they applied context awareness orientation. These researchers - [3, 14] had issues with classifying the ordered and (usually inevitable) unordered nature of the contact list and LoCTs in mobile phones. The challenge with using the social context and the context awareness orientation stems from the inability of mobile system to determine the Who, When, Where, What and How of a user. The cost implication of translating this resource to the benefit of users can also be huge. For instance, the phones of victims will need to work under complex and dynamic local and even Internet condition(s)[15]. Even a higher level of contexts awareness information (e.g., meeting, leisure, travelling) may be needed [15]. So, in an emergency situation, what of if a victim does not have a well equip phone? That is, a phone with a range of advanced sensors and accelerometer. Moreover, how many cell phones used by individuals (especially in Nigeria, where the majority leave in poverty) possess the foregoing type of feature? Even, if all the cell phones that are in use today in Nigeria are well equipped, what is the guarantee that in an emergency situation there will be network availability to allow rescuers use the phone? In the Nigeria context of today, where there are incessant network failures due to several factors; the question is, what will be the fate of victims in emergency situation; what of the rescuers who will be disquieted at the lack of assistance from the phone? This highlights the motivation to propose an emergency contact recommendation model that when implemented as an app (as done in this paper) can be used in any cell phone. The app will only need (at the barest minimum) the contact list of the phone, and/or the LoCTs (where they exist) to make its recommendation.

It is evident that emergency situation can be managed using technologies, such as chatting, calling and hanging out. Additionally, the aforementioned researchers did not address the issue of privacy, which can be debilitating. For instance, in an emergency situation, the amount and level of information that must be available should be unlimited. If a phone is picked at the point of 
accident and the phone is locked or the phone's security level is high, the contact phonebook cannot be accessed or the context aware information might not be available to take advantage of. Therefore, the need to grant access, not only to ICE contact list; the usual phone contact list; and LoCTs (at least any of these lists must be available) during emergency situation is overarching. Cognizance of the foregoing, the model cum app presented in this paper uses the leverage of Emergency Call (EC). EC is present in any mobile phone, especially when a phone is pass worded to forestall security. Thus, the ECRS uses the EC to use the ICE contact list (if available) or the usual phone contact list and/or LoCTs (if ICE is not available). Relying on this the ECRS can be used to make recommendation during emergencies. It is interesting to note that in all the works reviewed so far, the concept of ICE, group contacts - that are designated using words that connote close ties like "family", and keyword search were not properly addressed in developing their models. This paper therefore pursued the provision of an ECRM that can be adapted to meet the development of ECRS, which can work for all and sundry phones considering the peculiar condition of the average Nigerian user as earlier discussed.

\section{METHODS}

This section contains the methods in form of the techniques, approach and software technology that was applied in the formulation of the ECRM, the implementation and the testing of the ECRS that is presented in this paper. To present the foregoing this section is sectionalized into the section on model formulation and description, model implementation and testing.

\subsection{Model's Formulation and Description:}

The ECRM was formulated based on an architectural provision. This was necessary in order to highlight the contribution of this paper to the domain of emergency contact recommendation. The architecture was pieced together following what obtains in recommender system literature $[3,14,15]$. For the sake of validity; particularly of the purpose of the ECRM, the architecture as presented in Figure 1 shows three basic modules, namely the LP, P\&C, and Vis modules (see Figure 1). The P\&C module is made up of two submodules - the CIA and the Rec sub-modules (see Figure 1). The novel contribution of the work reported in this paper to emergency recommendation literature is the re-engineering of the CIA and the Rec modules. The contribution entails the integration of the technique of probabilism using the BNt, and the synergy of the Dustminer Algorithmic technique (DAt) and the Direct Discriminative pattern mining technique (DDPminet). In the emergency recommendation literature, the BNt was used to handle issues that bother on the optimization of probabilistic inference to unravel issues of uncertainty, and then utilize expert's pre-knowledge. However, how to handle the case of the existence of unordered contact list, which was initially a challenge was resolved with respect to emergency contact recommendation using the DDPminet. Very often, the contact list and LoCTs in users' phones are unordered. Even when ordered, the BNt does not classify them too well to be able to allow the ECRS make reasonable inference, talk less of when unordered. This challenge motivated the need to adopt and adapt the DAt, which made it possible to incorporate the DDPminet.

\subsubsection{The Bayesian Network Technique:}

The plausibility of the ECRM in this paper can be highlighted using the ECRS architecture. The input features that are shown in the LP module contribute to the robustness the ECRS architecture seeks in order to ensure the viable implementation of an ECRS. At the barest minimum (at least) any one of the input features LoCTs (Logs), ICE, Keyword, Av and Loc must be available for efficient recommendation to be made by the P\&C module (see Figure 1). However, the Loc feature (see Figure 1) is a critical deciding factor in a victim's Smartphone if there is a Global Positioning System (GPS) sensor for easy scanning of location.

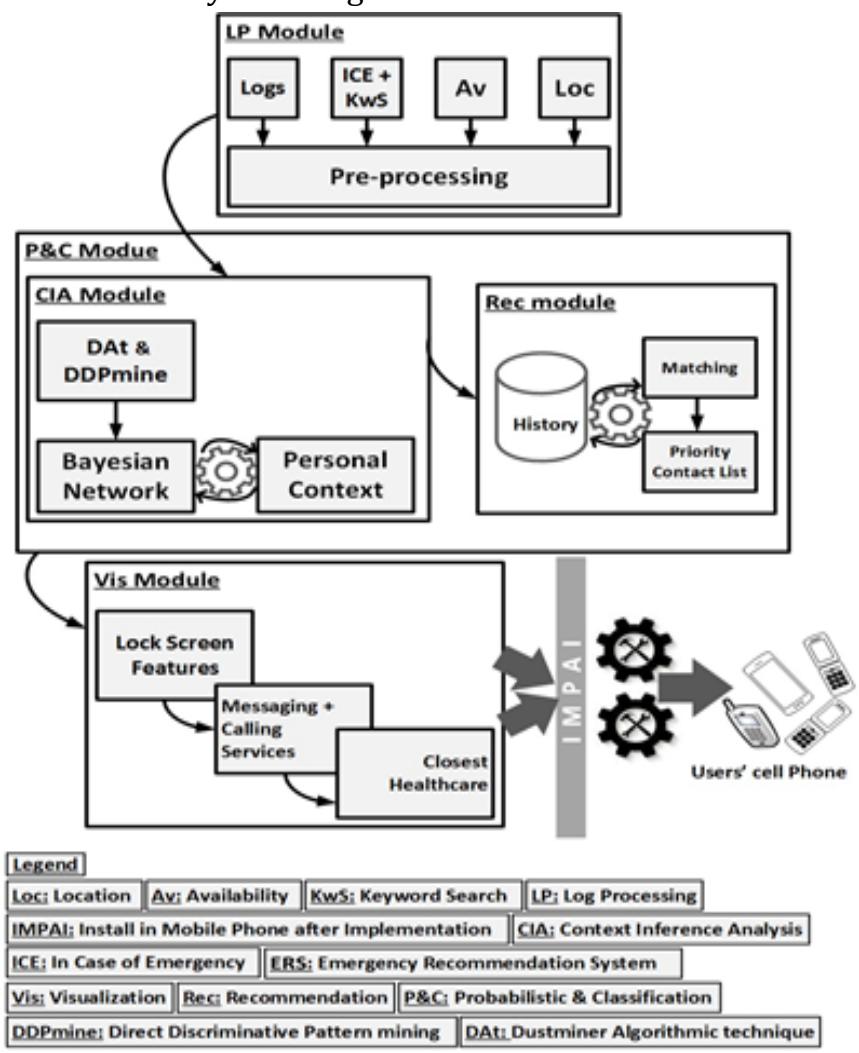

Figure 1: Emergency recommendation system architecture 
The location will be easily saved to a local server and can be accessed by the application once it is installed on a Smartphone. In existing systems, this factor requires that the other three factors are present and received as input. However, as earlier mentioned, considering the Nigeria context, at least only one of the input feature is enough to arrive at the recommendation of contacts. The DAt was employed to handle the challenge of lack of orderliness when all the input features are active or present in the LP module (see Figure 1). When all the input features (see Figure 1) are present and the GPS is enabled, the anticipated lack of orderliness becomes complex. The DDPminet was used to handle this complexity. In the P\&C module the BNt works in tandem with the personal context, which bothers on the individual user (a possible victim) way of organizing contact on his/her mobile phone. Based on this context, there is a scanning mechanism that works as a service that runs from time to time to generate good inference(s), which result is updated regularly on the server. For mobile phones with GPS capability proximity to a close relation (as a third party) is also handled as part of the personal context. To determine the feature of availability, the number of missed calls, the number of unread messages, and the status of a mobile phone connectivity was employed as parameters. This can also entail the last use of the mobile phone, which is checked in relation to the network provider(s) the user subscribes to. This information is always available on the server of the network the user subscribes to. From time to time the information will be accessed by the app when installed on a user's mobile phone. This information is used to determine who among the contact is available at the point at which an emergency situation occur.

The BNt provided the contrivance to make inference and generate the best contact using location and availability in relation to other parameters such as call logs, ICE contact (if any), and LoCTs gotten from an engaged Smartphone. The purpose of this combination was to be able to use the leverage of the possibilities provided by the input features based on the many relationships they present. For instance, it will be possible to scan the contact list of a victim's phone for names stored starting from the ICE as input into the pre-processing section (See Figure 1). If the victim had stored contacts on a group that is visibly identified as family, this will further make the policy of inferring to be better harnessed.

To make better inference on what contact to recommend in an emergency situation as the best contact recommendation some policies were formulated and followed based on the recommendation provided in [3]. Therefore, some of the policies include; relationships that reflect the concept of ICE, contact frequency, keyword contact search must be used for similarity matching. For example, how many times each contact in a victim's phone had called and was called by a victim were taken seriously and considered based on missed, dialled and received calls. For similarity matching a comparison is also necessary. Therefore, the last name mined from a victim's call profile with the ones on the contact list was carried out. Thus, any contact on the contact list that tallies attains greater probability of being a family member. For the contact frequency to be useful for inference making it must work in tandem with the contact span parameter. This is the total duration for each contact phone number that is dialled and the victim received call from. Common names such as dad, mum, papa, mama, sweet mum, sweet heart, precious, precious mum, and wife, which people often save their contact(s) with were taken advantage of in keyword contact search. A simple BNt was set up in the CIA module using the algorithmic approach presented in Figure 2. Based on the BNt the probabilistic inference from uncertainty and the use of expert's pre-knowledge was carried out following the practice in [3].

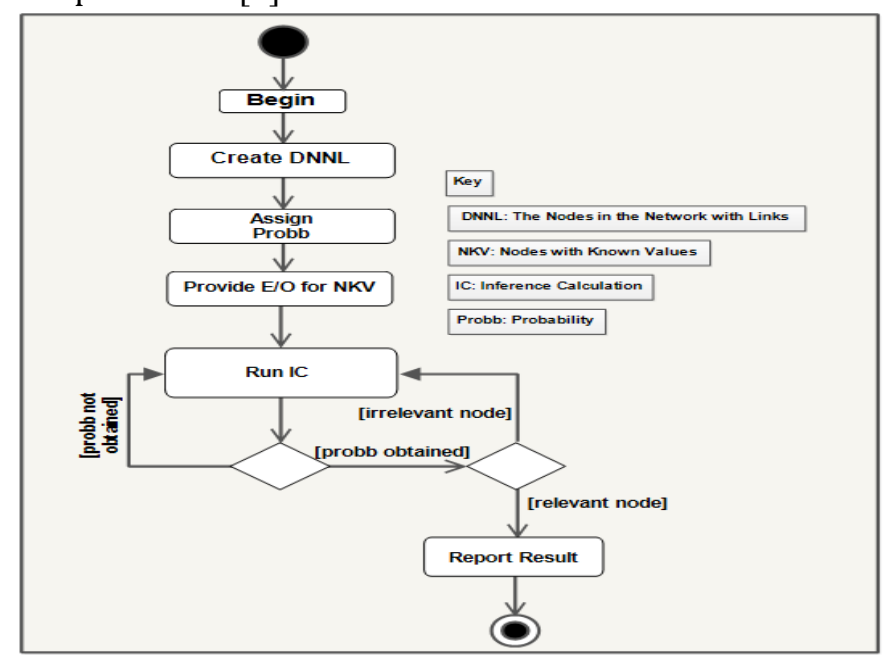

Figure 2: Algorithmic steps to create Bayesian Network

This expert pre-knowledge was codified as presented in Table 1. The foregoing pre-knowledge was elicited from the Bayesian Network operations in the CIA module as depicted in Figure 1. The BNt performs what is known as Bayesian Inference Network (BIN) operation. It is meant to recommend contacts of RFoCA. Formally, this is expressed as shown in Equation (1) as follows;

$$
\text { Let } B I N=G\left(B_{s}, \theta\right)
$$

In (1), $B_{s}$ is the network structure and $\theta$ is the Set of network parameters.

For easy formalization, we replace $\theta$ with $B_{\Phi}$ to be a set of network parameters as shown in Equation (1). This means that $B_{\Phi}$ stands for the conditional probabilities in Table 1, which are the probability of the set of network 
parameters $(\theta)$. For prior probability distribution $-P(\theta)$ of the set of network parameters, we use $B_{p}$ to represent its outcome. The motivation for $P(\theta)$ stems from the need to find the probability of the knowledge discovered. This knowledge is expert knowledge based on the history of relationships in the patterns inferred from the LoCTs, call log, contact list, third party information, and information from availability, proximity and status of a mobile phone connectivity, if the phone has a GPS. Since the knowledge discovered exist before inferring it; it means it is prior knowledge.

Table 1: Conditional probability table for Bayesian Network Tree

\begin{tabular}{|c|c|c|}
\hline Prob. & PoT & PoF \\
\hline $\mathrm{P}(\mathrm{Re})$ & 0.99 & 0.01 \\
\hline$P(A v)$ & 0.60 & 0.40 \\
\hline P (Loc) & 0.40 & 0.60 \\
\hline $\mathrm{P}(\mathrm{ICEc} \mid \mathrm{Re})$ & 0.99 & 0.01 \\
\hline$P\left(\mathrm{ICE} \mid \mathrm{Re}^{\mathrm{c}}\right)$ & 0.02 & 0.98 \\
\hline $\mathrm{P}(\mathrm{SM} \mid \mathrm{Re})$ & 0.70 & 0.30 \\
\hline $\mathrm{P}\left(\mathrm{SM} \mid \mathrm{Re}^{\mathrm{c}}\right)$ & 0.40 & 0.60 \\
\hline $\mathrm{P}(\mathrm{Kw} \mid \mathrm{Re})$ & 0.90 & 0.10 \\
\hline $\mathrm{P}\left(\mathrm{Kw} \mid \mathrm{Re}^{\mathrm{c}}\right)$ & 0.85 & 0.15 \\
\hline P (UMC|Av) & 0.60 & 0.40 \\
\hline $\mathrm{P}\left(\mathrm{UMC} \mid \mathrm{Av} \mathrm{v}^{\mathrm{c}}\right)$ & 0.20 & 0.80 \\
\hline P (UM|Av) & 0.70 & 0.30 \\
\hline$P\left(U M \mid A v^{c}\right)$ & 0.50 & 0.95 \\
\hline P (Last Dialled | Av) & 0.95 & 0.05 \\
\hline P (Last Dialled $\left.\mid A v^{c}\right)$ & 0.10 & 0.90 \\
\hline P (RecC| Closeness) & 0.60 & 0.40 \\
\hline $\mathrm{P}\left(\mathrm{Rec} C \mid\right.$ Closeness $\left.{ }^{c}\right)$ & 0.10 & 0.90 \\
\hline P (DiaC| Closeness) & 0.90 & 0.10 \\
\hline $\mathrm{P}\left(\right.$ DiaC| Closeness $\left.{ }^{c}\right)$ & 0.15 & 0.85 \\
\hline $\mathrm{P}(\mathrm{ConS} \mid \mathrm{Rec} C)$ & 0.80 & 0.20 \\
\hline $\mathrm{P}\left(\mathrm{ConS} \mid \mathrm{RecC}^{\mathrm{c}}\right)$ & 0.10 & 0.90 \\
\hline P (ConFrq| RecC) & 0.95 & 0.05 \\
\hline P (ConFrql RecCc) & 0.20 & 0.80 \\
\hline $\mathrm{P}(\mathrm{ConS} 2 \mid \mathrm{DiaC})$ & 0.90 & 0.10 \\
\hline $\mathrm{P}\left(\mathrm{ConS} 2 \mid \mathrm{DiaC}^{\mathrm{c}}\right)$ & 0.05 & 0.95 \\
\hline P (ConFrq2| DiaC) & 0.98 & 0.02 \\
\hline P (ConFrq2| DiaCc) & 0.10 & 0.90 \\
\hline $\mathrm{P}($ Closeness | Re) & 0.85 & 0.15 \\
\hline $\mathrm{P}\left(\right.$ Closeness | $\left.\operatorname{Re}^{c}\right)$ & 0.01 & 0.99 \\
\hline P (ICEno | Re, Av, Loc) & 0.99 & 0.01 \\
\hline P (ICEno | $\operatorname{Re}^{c}, A v$, Loc) & 0.20 & 0.80 \\
\hline P (ICEno | Re, Av c, Loc) & 0.70 & 0.30 \\
\hline P (ICEno | Re, Av, Loc c) & 0.80 & 0.20 \\
\hline P (ICEno | $\operatorname{Re}^{c}, A^{c}$, Loc) & 0.01 & 0.99 \\
\hline P (ICEno | Rec $\left., A v, \operatorname{Loc}^{c}\right)$ & 0.20 & 0.80 \\
\hline P (ICEno | Re, $\left.A v^{c}, \operatorname{Loc}^{c}\right)$ & 0.70 & 0.30 \\
\hline $\mathrm{P}\left(\right.$ ICEno $\left.\mid \mathrm{Re}^{\mathrm{c}}, \mathrm{Av}^{\mathrm{c}}, \mathrm{Loc}^{\mathrm{c}}\right)$ & 0.01 & 0.99 \\
\hline
\end{tabular}

Legend: ICEcontact (ICEc); Similarity Match (SM); Keyword (Kw); Unchecked Missed Calls (UMC); Unread Message (UM);

Relationship (Re); Availability (Av); Location (Loc); Received Calls (RecC); Probability of False (PoF) Contact Frequency (ConFrq); Probability (Prob); Probability of False (PoF); Probability (Prob.) Dialled Calls (DiaC); Contact Span (ConS); $\mathbf{X}^{\mathbf{C}}=\mathrm{X}$ contact; Probability of Truth $(\mathbf{P o T})$; Probability (Prob.)

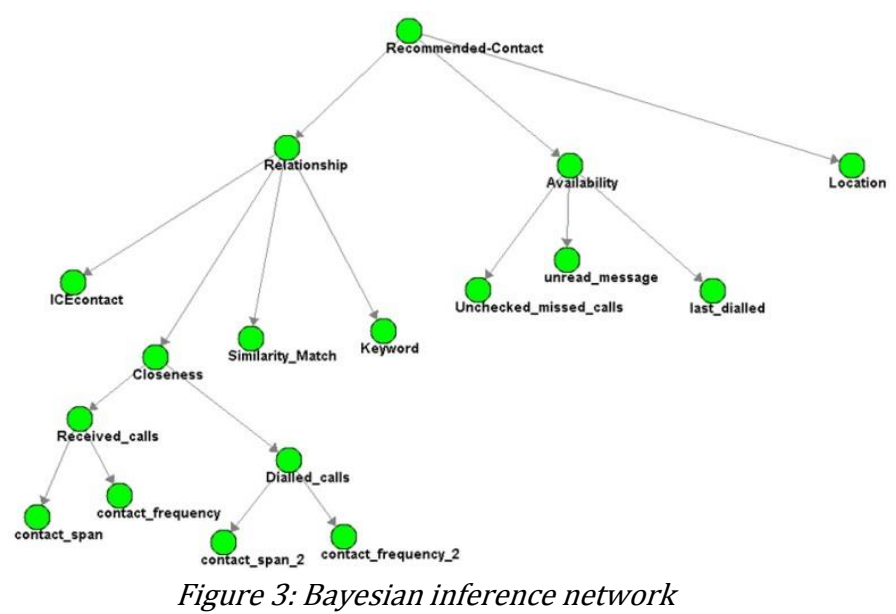

Therefore, the prior probability distribution - $P(\theta)$ of the process of discovering the knowledge - following [14, 3], was modelled and formally specified as presented in Equation (2) as follows;

$$
\begin{aligned}
& P\left(Z_{T}, Y_{T}, \theta\right) \\
& =P\left(Y_{T} \mid Z_{T}, B_{\phi}\right) P\left(Z_{T} \mid B p\right)
\end{aligned}
$$

Where $Z_{T}=\left\{z_{1}, z_{2}, \ldots, z_{T}\right\}$ - the set of representative state variables, $Y_{T}$ is the corresponding observations; $\mathrm{B}_{\Phi}$ is the conditional probability; and $B_{p}$ is the prior probability distribution.

It is interesting to state that $B_{s}$ and $\theta$ are based on the domain knowledge depicted in the LP module (see Figure 1) as prescribed in [3]. This resulted in the Network patterns depicted in Figure 3, which was leveraged in the CIA module for inferring emergency contact recommendation. The values used in the CIA module were assigned values from the pre-processing activities in the LP module. Priority in emergency recommendation is often taken seriously. This is because it is the degree of relationship that is extensible using keyword search, ICE contacts, and other contact groups. When the degree of relationship among contact is considered, the availability of contact persons becomes the second most important factor to determine the mining direction followed lastly by location. Interestingly, it is not in all cases, the closest contact is really the closest person to a victim. In order to verify this, it was important to get every information about the closest. Contact to a victim from the link of a closer contact following the links in the BIN as presented in Figure 3.

\subsubsection{The Dustminer Algorithmic and DDPmine Technique:}

These techniques were employed synergistically. The DDPminet approach was meant to tackle efficiency issues [16]. They arise from the inability of the BNt to handle events. In this work events are assumed as LoCTs, 
contact lists, and information from other parameters that can also be utilized at the LP module should a phone have GPS capability. The DDPminet was used to also achieve orders of magnitude speedup without any downgrade of classification accuracy [16]. Both the BNt and the DAt cannot handle the classification of events when they are unordered. The DDPmine was used to handle the lack of orderliness of events. The nondeterministic nature of events motivated the use of the DAt.

This nature arises because of unexpected or adverse distributed interactions between multiple and seemingly individually-correct components [17]. The owners of the contacts on victims' phone and their transactions epitomized this.

The issue of scalability also led to the adoption of the DAt. Scalability is a challenge in sequence mining for analysing logs to identify latent patterns that will be potentially useful. The number of combinations of the number of candidate patterns in a log do grow very quickly and exponentially too. After data mining the number of patterns returned can be in the order of several thousands [17]. For instance, the patterns that can be deduced from the network in Figure 3 can grow exponentially. This growth is inevitable. This is because each of the nodes has the potential to spurn any number of node(s) as shown in Figure 3. The main technique the DAt approach offered to this work is the ability to log many different types of events from the LP module and then analyse the logs in an automated fashion to extract the sequences of events. Since it cannot present the events in ordered form, but having taken care of the nondeterministic nature, the DDPminet takes over. It then takes care of the frequent unordered set of items events, and present the "culprit sequence". It also prunes the patterns detected as early as possible without risking the possibility of dropping the "culprit" sequence. In this paper the "culprit" sequence is the list of contacts that is adjudged as the possible list of contacts that are closest to a victim in an emergency situation. The DAt performs a discriminative analysis to arrive at this. The BNt takes the "culprit sequence" and performs the function of inferring the best contact to be recommended by the Rec module (see Figure 1). This is done considering the history of the victim (in question) using the matching technique as provided in the architecture. Both the DAt and the DDPminet are algorithmic in nature. The synergize form of the algorithmic model showing the DAt and the introduced DDPmine technique in pseudo code is presented in Figure 4.

\section{RESULT AND DISCUSSION}

Following the experimental testing standard in [14], the ECRS app was tested for both suitability and usability. Accuracy metric was used to test the suitability of the system.

Algorithm: DAt in synergy with the DDPminet

Input: Set of events as Good Logs (eGL), set of events as Bad Logs (eBL) Output: Set of discriminative sequences of events

1. $S_{\text {common }}=\epsilon, \operatorname{SeGL}=\in, \operatorname{SeBL}=\epsilon, I=1$

2. while $(\mathrm{SeGL}==\in$ or $\mathrm{SeBL}==\epsilon$ )

2.1 SeGood= GenerateFrequentSubSequences(eGL, I, Scommon)

2.2 SeBad= GenerateFrequentSubSequences(eBL, I, Scommon)

2.3 If (eGL, I, Scommon = ordered) GenerateOrderedList (eGL, I, , Scommon)

2.4 If (eBL, I, Scommon $=\urcorner$ ordered) GenerateOrderedList (eBL, I, Scommon)

2.5 If (SeGL $==\in$ ) SeGL = SeGL U FindDiscriminative(SeGood, SeBad)

2.6 If $(\mathrm{SeBL}==\in)$ SeBL $=$ SeBL U FindDiscriminative(SeBad, SeGood)

$2.7 \mathrm{~S}_{\text {common }}=$ FindCommon(SeGL, SeBL) $2.8 \mathrm{i}=\mathrm{i}+1$

Function: GenerateOrderedList

Input: Set of eGL as FP-tree $P$, min_sup $s$, \&Set of eBL as FP-tree $Q$, min_sup s,

Output: Set of ordered sequence of events (ordered_eGL, ordered_eBL)

Procedure DDPmine $(P, Q, s)$

1. If $P=\in, \& Q=\epsilon$

2. return;

else;

3. $a_{1}:=$ branch_\&_bound $(P, s$, null);

$\mathrm{a}_{2}:=$ branch_\&_bound $(Q, s$, null $)$;

4. Compute the transaction id list $T\left(\mathrm{a}_{1}, \mathrm{a}_{2}\right)$ containing $\mathrm{a}$;

5. $P^{\prime}:=$ update_tree $\left(P, T\left(a_{1}\right)\right)$;

$Q^{\prime}:=$ update_tree $\left(Q, T\left(a_{2}\right)\right)$;

6. ordered_eGL, eBL:= $\left\{a_{1}, a_{2}\right\} \cup \operatorname{DDPmine}\left(P^{\prime}, Q^{\prime}, s\right)$

7. returnordered_eGL, $e B L$

Function: FindDiscriminative

Input: Set of Frequent SubSequences(A),Set of Frequent SubSequences $(B)$

Return: Set of discriminative SubSequences that distinguishes $A$ from $B$

Assumption: Each sequence $p_{i}$ in $A$ or B has two supports, supinfileandsupiacrossfile

supinfilerecords the average number of occurrence of $p_{i}$ within a file.

sup $_{\text {iacrossfile }}$ records the probability of occurrence of $p_{i}$ in a file.

1. S discriminative $=\epsilon$

2. for each sequence $p_{i}$ in $A$

2.1 for each sequence $q_{j}$ in $B$

2.1.1 if $\left(p_{i}==q_{j}\right)$ then

if(supiacrossfile $/$ sup $_{\text {jacrossfile }}<$ ) then $S_{\text {discriminative }}=S_{\text {discriminative }} \cup p$

else if(supiinfile/supjinfile__) then $S_{\text {discriminative }}=S_{\text {discriminative }} U p_{i}$

3. Return Sdiscriminative

Function: GenerateFrequentSubSequences

Input: Set of Logs $(L)$,sequenceLength $(K)$, baseSet $\left(S_{\text {common }}\right)$

Return: Set of frequent SubSequences of length $\mathrm{K}$

1. Use the Apriori algorithm to generate frequent subsequences of length $K$ using

baseSet

2. Return frequent subsequences of length $\mathrm{K}$ generated at step 1

Figure 4: The synergize algorithmic technique 
In order to ascertain the ease with which users would use the system to their advantage, the system was tested using usability metric. For accuracy, the ratio of the number of correct recommendation(s) to the total number of recommendation(s) was adopted. This ratio was validated by comparing callee's recommendation with user's choice of emergency contact. Based on the results gotten, it was observed that three (3) recommendations were mostly appropriate. Though, at some other time four (4) recommendations were found to be appropriate out of five (5) selected best contacts. The accuracy of the results range between $5 \%$ to $70 \%$.

This happened when the callees where made the candidates for each recommendation. It was also discovered that the topmost person on the recommended list had the closest personal relationship. However, lack of sufficient logs to do proper testing presented some setback, which need to be improved upon.

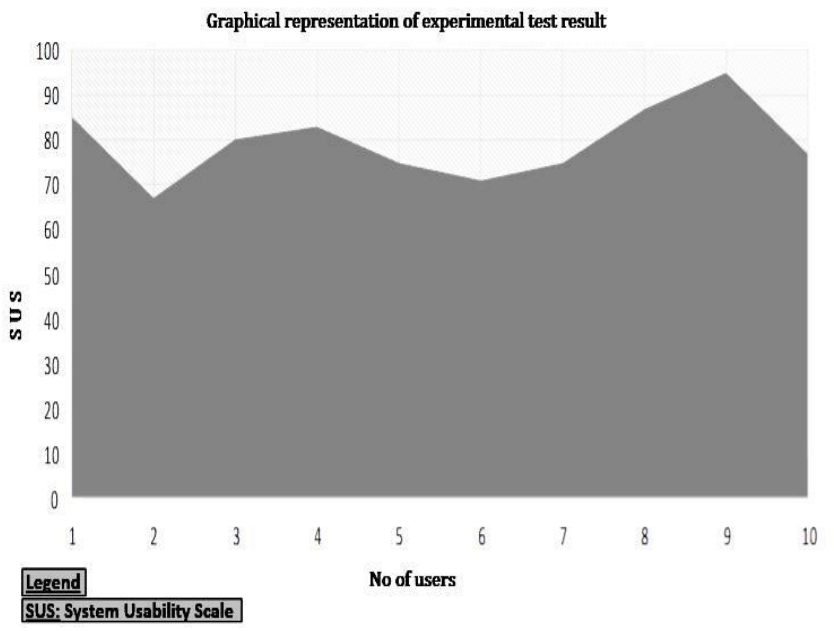

Figure 5: Experimental test result of system usability

Following the standard in literature, the System Usability Scale (SUS) questionnaire were employed as practiced in [18] and [14]. The SUS - a ten-item scale was employed to present a global view of what using the ECRS app is like among ten users. It allowed the subjective

assessments of the usability of the ECRS app. To be consistent with what obtains in literature $[19,14]$, the testing adopted the de facto score range - which is from 0 to 100 - and the SUS questions (in modified form see Table 2). The result of the analysis of the cognitive data elicited is presented graphically as shown in Figure 5.

The ECRS was implemented using Java development and android tool kit. The app ran successfully on the smartphone with android mobile Operating System (OS). It can also run on any android platform OS from 4.2.2 Jelly Beans upwards. The android operating system, the eclipse IDE (for building the application interfaces), and the online server for the database management form the software requirements for the implementation of the ECRM to the ECRS. As expected the online server of the network a victim's mobile phone is subscribed to serves as the database management server where preprocessed logs are stored. This awaits the use of the app in the event that there is an emergency situation. For the foregoing server and mobile phone - client - to communicate with each other a TCP/IP socket was introduced into the app.

Table 2: The adopted and modified SUS Questionnaire

\begin{tabular}{|c|c|}
\hline $\mathrm{S} / \mathrm{N}$ & SUS Questions \\
\hline 1. & I think I would like to use the ECRS app. Frequently \\
\hline 2 & I found the ECRS app. unnecessarily complex \\
\hline 3 & I thought the app. was easy to use \\
\hline 4. & $\begin{array}{l}\text { I think that I would need the support of a technical } \\
\text { person to be able to use the ECRS app. }\end{array}$ \\
\hline 5. & $\begin{array}{l}\text { I found the various functions in the ECRS app. very } \\
\text { well integrated }\end{array}$ \\
\hline 6. & $\begin{array}{l}\text { I thought there was too much inconsistency in this } \\
\text { application }\end{array}$ \\
\hline 7. & $\begin{array}{l}\text { I would imagine that most people would learn to use } \\
\text { the ECRS app. very quickly }\end{array}$ \\
\hline 8. & I found the ECRS app. very cumbersome to use \\
\hline 9. & I felt very confident using the ECRS app. \\
\hline 10. & $\begin{array}{l}\text { I needed to learn a lot of things before I could get going } \\
\text { with the ECRS app. }\end{array}$ \\
\hline & $\begin{array}{l}\text { Legend: SUS: System Usability Scale; ECRS: Emergency } \\
\text { Contact Recommendation System; app: Application; } \\
\text { S/N:Serial Number }\end{array}$ \\
\hline
\end{tabular}

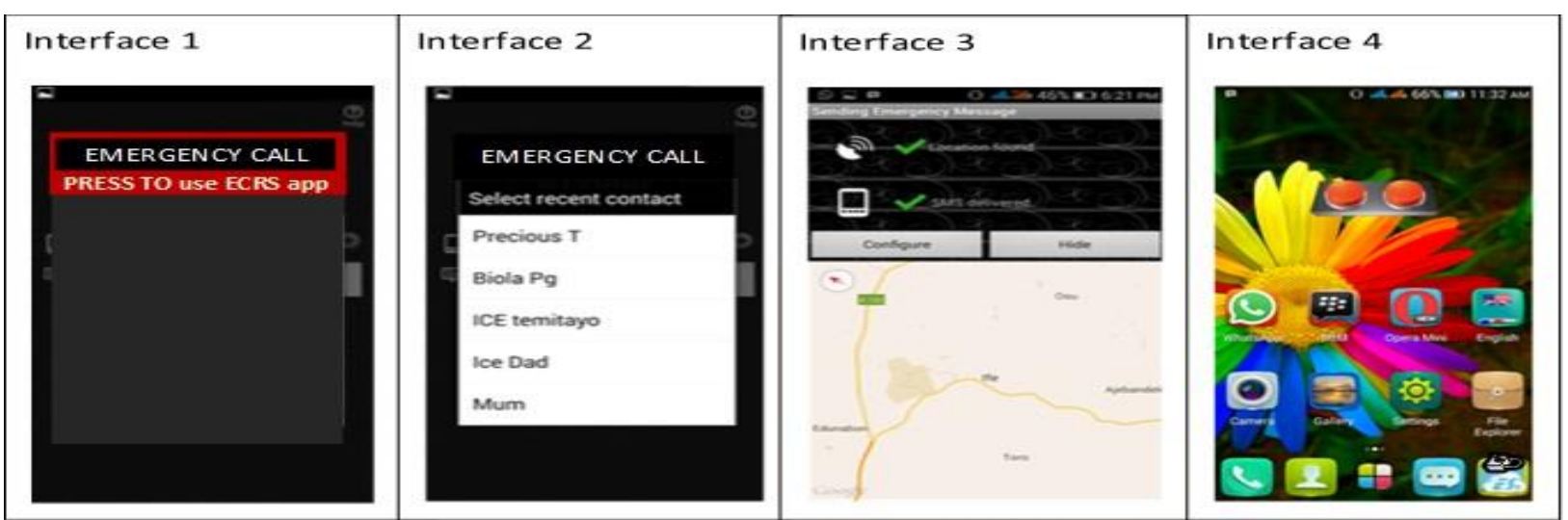

Figure 6: The ERS interface for emergency Contact Recommendation 
The system operates using the windows area where a priority contact list is displayed (see interface 2 in Figure 6). This happens after the decision made using the BNt in synergy with the DAt and DDPmine technique considering the various factors discussed earlier. On installing the app in the phone of the users whose phones were used for the alpha testing; it was discovered that if a phone is pass worded, Interface 1 comes handy. A click on the emergency call provides the option to use the app. On clicking the app, the app recommends the best contact to call in order of closeness; that is the closest family or acquaintance of the victim. The Interface 2 shows five of such recommendations. Once any of the five contacts is selected, and the message button is pressed, Interface 3 is opened up.

This third window gets the location information of the victim(s) automatically, and also provides a message status, auto-suggestion message features and display a map. This shows the direction to any place the user is directed to in order to help the victim seek medical help (see Figure 6). Interface 4 comes handy when there is not security details in any phone that has the app installed. The user simply dials the ECRS widget - the icon with a key symbol on top of it (see the right hand side of the bottom of the Interface 4 of Figure 6).

From the graph result presented in Figure 5, it is clear that the 10 users who used the system had SUS score of from $67 \%$ and above. This score indicates that the Vis module (see Figure 1) was able to assists the 10 users to perform the functionality that pertains to lock screen features. That is the ECRS app was able to help some of the users of the ECRS app to bypass the security check on users' phone (using EC) to a commendable extent in order to make emergency contact. The results (see Figures 5 and 6) also imply that the messaging and calling services provided by the app was consistent with what is expected in literature [17]. The results also support the fact that the app was able to indicate the location of victims as shown in Figure 6.

\section{CONCLUSION}

The aim of this paper was to present a model approach to the development of emergency contact recommendation. The paper extended the concept of ICE as theorized by Bob Brotchie [6]. It therefore formulated a model with probabilistic data mining and classification properties. The paper employed an architectural approach (Figure 1) to highlight its contributions. The plausibility of the ECRS mobile app presented in this paper is in its ability to assist users to (i) identify the relationships between contacts, (ii) get the location status of victims and display it (if GPS is available on the victims' phone) (see
Interface 3 of Figure 6), (iii) pinpoint contact proximity, and (iv) recommend useful contact(s) (see Interface 2 of Figure 6). The foregoing possibilities were tested and the best contact recommendation is as presented in Interface 2 of Figure 6.

In future, we intend to find out if the algorithms employed in this work satisfy basic time complexity requirement(s), and also test them on real life emergency scenarios with more users. The approach used to test the ECRS was limited to only alpha testing. It was tested among users that are familiar with apps operation. It will also be interesting to ensure that the ECRS app is able to suggest the location of the nearest healthcare facility to an emergency scene.

\section{REFERENCES}

[1] Cui, X., Baker, J. M., Liu, N., and Reiss, A. L. "Sensitivity of fNIRS Measurement to Head Motion: An Applied Use of Smartphones in the Lab". Journal of neuroscience methods, 245, pp. 37-43, (2015).

[2] Experian Marketing Services (EMS). "Mobile Measurement: A White Paper for Marketing that Keeps Pace with Tablets and Smartphones": pp. 1-6. Retrieved from http://www.experian.co .uk/assets/marketingservices/white-papers/wp-hitwise-mobilemeasurement.pdf @ 21: 27pm on August 05, (2015).

[3] Min J.-K. \& Cho S.-B.Mobile Human Network Management and Recommendation by Probabilistic Social Mining. IEEE Transactions on Systems, Man, and Cybernetics, 41 (3), pp. 444-451, (2011)

[4] Ejidokun, A. O., Akhigbe, B. I., Aderibigbe, S. O., and Afolabi, B. S. "A Probabilistic Model for Emergency Contact Recommendation", In the Proceedings of $O A U$ TekCONF, Vol. 5, pp. 277-281, (2015).

[5] Solagberu, B. A., Ofoegbu, C. K., Abdur-Rahman, L. O., Adekanye, A. O., Udoffa, U. S., and Taiwo, J. "Pre-hospital Care in Nigeria: A Country without Emergency Services". Nigerian Journal of Clinical Practices, 12 (1), pp. 29-33 (2009).

[6] Riccitello, J. M. (2008). "ICE Awareness Among Local EMS Personnel". In the University of Texas Medical Branch Graduate School of Biomedical Sciences retrieved from https://repositories.tdl.org @ 8:00am on July 31 st, (2015).

[7] OSU, "UNIOSUN, In Case of Emergency". Retrieved from https://www.uniosun.edu.ng @11:00am on August 10, (2014).

[8] Wei, K., Huang, J., Fu, S. "A Survey of E-Commerce Recommender Systems". In Proceedings of the International Conference on Service Systems and Service Systems and Service Management, USA, IEEE, pp. 1-5, (2007).

[9] Hand, D. J., Mannila, H., and Smyth, P. "Principles of Data Mining”. Cambridge, Massachusetts, USA: MIT press, (2001). 
[10] Milewski, A. E., and Smith, T. M. "Providing Presence Cues to Telephone Users". In Proceedings of the ACM Conference on Computer Supported Cooperative Work (CSCW), Philadelphia, pp. 89-96, (2000).

[11] Schmidt, A., Takaluoma, A., and Mantyjarvi, J. "Contextaware Telephony Over WAP", Personal Technologies, 4(4), pp. 225-229, (2000)

[12] Tang, J. C., Yankelovich, N., Begole, J., Van Kleek, M., Li, F., and Bhalodia J. "ConNexus to Awarenex: Extending Awareness to Mobile Users". In Proceedings of the SIGCHI Conference on Human Factors in Computing Systems (CHI), pp.221-228, (2001).

[13] Hwang, R. H., Tsai, S. Y., and Wang, C. Y. "UbiPhone: Human-centered Ubiquitous Phone System". IEEE Pervasive Computing, (2), 40-47, (2009).

[14] Min, J.-K., Jang, S.-H., and Cho, S.-B. "Mining and Visualizing Mobile Social Network Based on Bayesian Probabilistic Model". In D. Zhang et al. (Eds.): UIC 09', Springer LNCS 5585, pp. 111-120, (2009).

[15] Wei, Z., Deng, R. H., Shen, J., Zhu, J., Ouyang, K., and Wu, Y., Multidimensional Context Awareness in Mobile Devices. In Multimedia Modelling: pp. 38-49, Springer. (2015).

[16] Cheng, H., Yan, X., Han, J., and Yu, P. S. "Direct Discriminative Pattern Mining for Effective Classification". In Proceedings of IEEE 24th International Conference on Data Engineering (ICDE) 2008. (pp. 169178). (2008).

[17] Khan, M. M. H., Le, H. K., Ahmadi, H., Abdelzaher, T. F., and Han, J. "Troubleshooting Interactive Complexity Bugs in Wireless Sensor Networks using Data Mining Techniques.
ACM Transactions on Sensor Networks, 10(2), pp. 31-65, (2014).

[18] Bangor A., Kortum, P.T., and Miller J.T. "An Empirical Evaluation of the System Usability Scale". International Journal for Human-Computer Interaction, 24 (6), pp.574594, (2008).

[19] Brooke, J. "SUS: A Quick and Dirty Usability Scale". In Jordan P.W., et al. (eds.), Usability Evaluation in Industry. London: Taylor and Francis, (1996).

[20] Oulasvirta, A., Petit, R., Raento, M., and Tiita, S "Interpreting and Acting on Mobile Awareness Cues". In Human-computer Interaction, 22 (112), pp. 97-135, (2007).

[21] Madueme, T. C., and Wokoro, P. G. "The Use of Artificial Neural Networks in the Theoretical Investigation of Faults in Transmission Lines", Nigerian Journal of Technology, Vol. 34 Number 4, pp.851 - 860, (2015).

[22] Udeh, C. N. “Irrigation Management Decision Model Using Probabilistic Hydrologic and Irrigation Efficiency Parameters", Nigerian Journal of Technology, Vol. 4, Number 1, pp. 29-42, (1980).

[23] Correa, M., Bielza, C., and Pamies-Teixeira, J. "Comparison of Bayesian Networks and Artificial Neural Networks for Quality Detection in a Machining Process". Expert Systems with Applications, Vol. 36, number 3, pp. 72707279, (2009).

[24] Zhuang, X., Sun, Y., \& Wei, K. "SMoCoR: A Smart Mobile Contact Recommender Based on Smart Phone Data". In Computer Software and Applications Conference (COMPSAC), 2014 IEEE 38th Annual(pp. 634-635), (2014). 